\title{
Correction to: Intellectual Disability and Social Policies of Inclusion
}

\section{Correction to:}

D. P. Treanor, Intellectual Disability and Social Policies of Inclusion, https://doi.org/10.1007/978-981-13-7056-4

In the copyright page, the author affiliation was erroneously mentioned as Hobart, NSW. It has been corrected as Hobart, TAS.

The updated online version of this book can be found at https://doi.org/10.1007/978-981-13-7056-4 